\title{
Robotic zero ischemia partial nephrectomy: step by step surgical technique with tips and tricks
}

This article was published in the following Dove Press journal:

Robotic Surgery: Research and Reviews

17 March 2014

Number of times this article has been viewed

\author{
Abdullah Erdem Canda' \\ Mevlana Derya Balbay ${ }^{2}$ \\ 'Yildirim Beyazit University, School \\ of Medicine, Ankara Ataturk Training \\ and Research Hospital, Department \\ of Urology, Ankara, ${ }^{2}$ Memorial Sisli \\ Hospital, Department of Urology, \\ Istanbul, Turkey
}

\begin{abstract}
Currently, most renal cell carcinoma cases are detected incidentally as small renal masses during radiologic imaging, such as abdominal ultrasound or computed tomography, and partial nephrectomy is being increasingly performed for these lesions. There has been a worldwide increase in minimally invasive surgical approaches, and robotic partial nephrectomy (RPN) in particular has the advantages of more rapid tissue dissection, reconstruction, intracorporeal suturing, three-dimensional optical magnification, dexterity in motion, and ability to perform tremor-free and delicate movements with three independent robotic arms. Renal hilar clamping during RPN decreases bleeding, but warm ischemia might negatively impact renal function. However, with sufficient surgical experience in robotic kidney surgery, zero ischemic or off-clamp RPN might be a safe and feasible minimally invasive surgical approach that has the advantage of avoiding complete renal ischemia, thus avoiding a decrease in renal function particularly in small, less complex, peripherally located, exophytic renal masses less than 3 $\mathrm{cm}$ in size. Currently available absorbable barbed sutures could further facilitate performing internal and external renorraphy, as could fibrin and tissue sealants that may also be used for hemostasis. This approach might be particularly important in patients with underlying kidney disease.
\end{abstract}

Keywords: robotic partial nephrectomy, zero ischemia, renal cell carcinoma, small renal masses, minimally invasive surgery, surgical technique

\section{Introduction}

Currently, most cases of renal cell carcinoma are detected incidentally as nonmetastatic small renal masses, and nephron-sparing surgery is regarded as the surgical treatment. Minimally invasive surgical approaches, including laparoscopic and in particular robot-assisted partial nephrectomy (RPN), rather than open surgery are increasingly being performed for small renal masses. ${ }^{2}$

Temporary renal artery clamping or renal artery and renal vein clamping is done before starting excision of the renal lesion in order to decrease bleeding and to provide a bloodless and clear image for tissue dissection during RPN. ${ }^{3}$ However, hilar clamping cuts off the blood supply to the entire kidney, which may result in decreased renal function, which is a particular problem in patients with underlying chronic kidney disease. ${ }^{3}$

The number of papers on the topic of zero ischemia RPN is increasing in the literature. Herein, we describe our technique for zero ischemia RPN in the management of small renal masses, and present a series of surgical robotic images of procedures performed by the authors.
Correspondence: Abdullah Erdem Canda Department of Urology, Yildirim Beyazit University, School of Medicine, Ankara Ataturk Training and Research Hospital, Bilkent 06800, Ankara, Turkey

Tel +905067635466

$\mathrm{Fax}+903122912525$

Email erdemcanda@yahoo.com 


\section{Patient selection}

In line with our current practice, patients with small renal masses are considered for zero ischemia RPN. If an accessory lower pole artery is present and the tumor is located peripherally as an exophytic mass lesion $<3 \mathrm{~cm}$ in size, we selectively occlude the lower pole artery by applying a laparoscopic bulldog clamp. Then, in the presence of an accessory artery selectively supplying the renal mass, we apply a polymer ligation clip (Weck ${ }^{\circledR}$ Hem-o-lok ${ }^{\circledR}$; Teleflex Medical, Research Triangle Park, NC, USA) to block the blood supply to the mass and perform zero ischemia RPN. In patients with a solitary kidney and in those with the tumor characteristics described above, zero ischemia RPN could be performed that might have a positive impact on residual postoperative kidney function. Another important tool that might be required during this type of surgery is intraoperative endoscopic ultrasound, which might be helpful for showing the depth of the tumor before starting excision. We currently do not attempt to perform zero ischemia RPN for larger-sized tumors or if intraoperative endoscopic ultrasound is not available.

\section{Port sites}

Following general anesthesia, the patient is positioned in the $60^{\circ}$ lateral decubitus position. An open trocar insertion technique is used. The location of the ports is similar to that used in the robotic pyeloplasty procedure reported by us elsewhere (Figure 1). ${ }^{4}$ A $12 \mathrm{~mm}$-sized port for a threedimensional lens is introduced into the abdomen and located lateral to the rectus abdominis muscle above the umbilicus. Intra-abdominal pressure of $15 \mathrm{mmHg} \mathrm{CO}$ is then applied and a $0^{\circ}$ three-dimensional robotic lens is introduced into the abdomen. An $8 \mathrm{~mm}$ robotic port is also introduced under direct vision $8 \mathrm{~cm}$ away from the camera port and located close to the anterior superior iliac spine. A second $8 \mathrm{~mm}$-sized

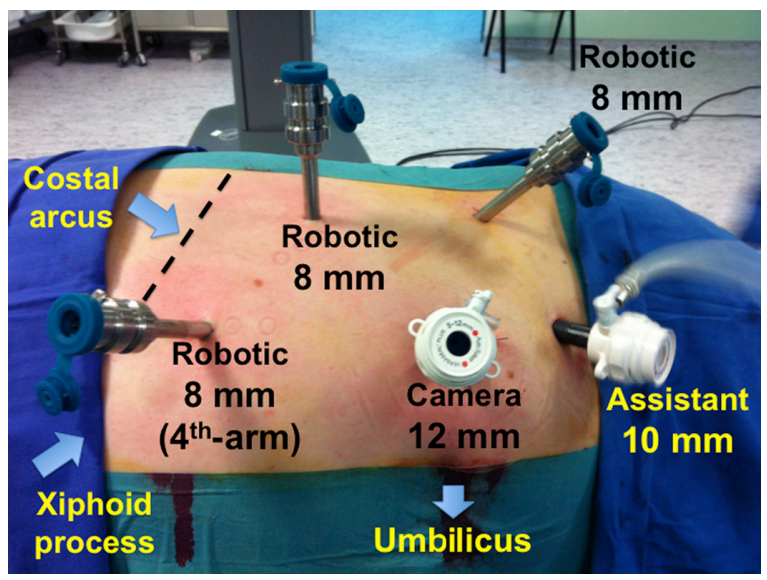

Figure I Abdominal port sites (left side). robotic port is introduced under direct vision and located approximately $8 \mathrm{~cm}$ away from the camera port close to the costal arcus on the mid clavicular line. A $12 \mathrm{~mm}$-sized assistant port is inserted into the abdomen and located $4-5 \mathrm{~cm}$ lateral to the camera port for the bedside assistant surgeon in order to introduce endoclips, sutures, suction, vascular tape, hemostatic agents, and the endobag. In some cases, we use the fourth robotic arm for further tissue retraction and assistance that might facilitate the procedure. Although using the fourth robotic arm might increase the cost of the surgery, particularly in reconstructive procedures such as RPN, it is still useful to have the fourth arm of the Da Vinci surgical robot available to assist the console surgeon. However, if the port for the fourth arm is not appropriately inserted, the arms may crash outside the abdomen following docking of the surgical robot. To avoid this, we dock the patient cart at an angle of $15^{\circ}-20^{\circ}$ cephalad over the patient's shoulder to accommodate the natural position of the kidney.

\section{Robotic and endoscopic instruments}

Monopolar $8 \mathrm{~mm}$ Maryland curved scissors, bipolar $8 \mathrm{~mm}$ Maryland forceps, and a robotic needle holder are used. If the fourth arm is used, ProGrasp ${ }^{\circledR}$ (Intuitive Surgical, Sunnyvale, CA, USA) robotic forceps are also used. Endoscopic suction is used for irrigation and removing blood and smoke. Additionally, an endoscopic needle holder is introduced to remove suture materials, along with an endoclips applier to introduce endoclips into the abdominal cavity.

\section{Surgical technique}

An edited video of robotic zero ischemia partial nephrectomy performed by Dr Canda can be watched here (supplementary video). The white line of Toldt is identified, the colon is

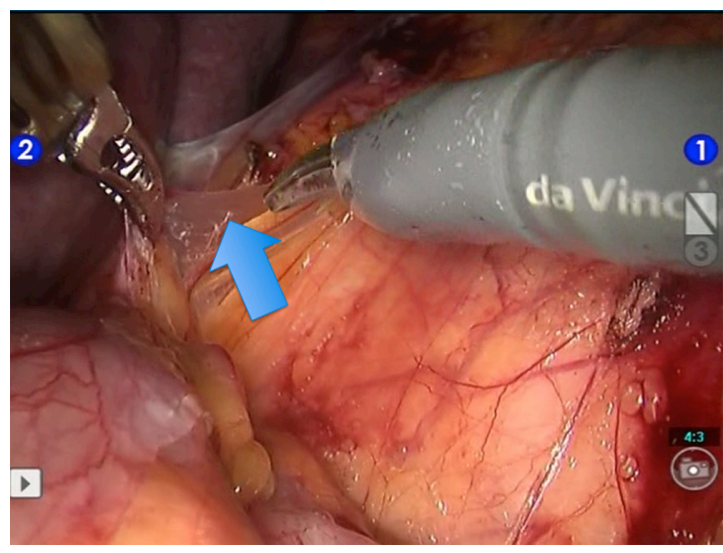

Figure $\mathbf{2}$ Identification and incision of the white line of Toldt and mobilization of the colon and access to the retroperitoneal space (arrow). 


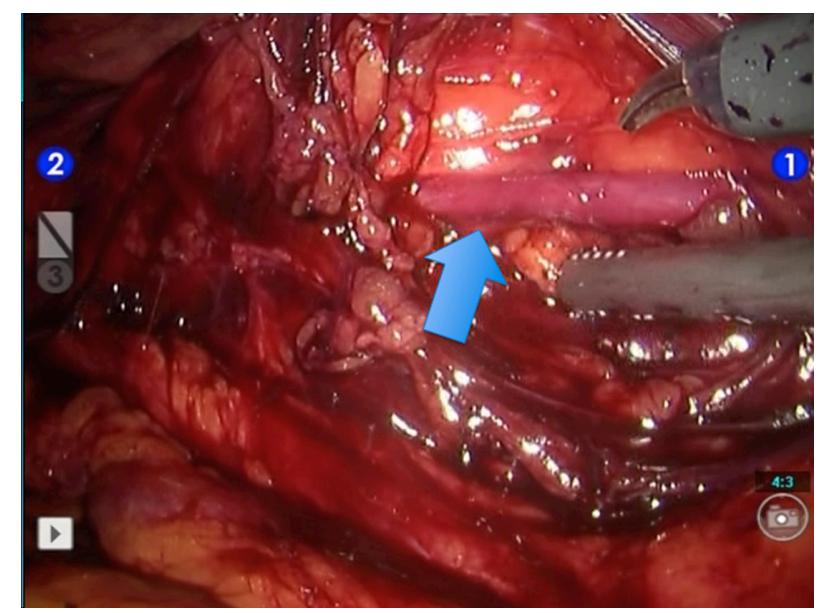

Figure 3 Identification of the ureter in the retroperitoneal area (arrow).

mobilized medially, and the retroperitoneal space is entered (Figure 2). The ureter is identified in the retroperitoneum (Figure 3) and followed up to the renal hilum, where the renal vessels are identified. The renal vein (Figure $4 \mathrm{~A}$ and $\mathrm{B}$ ) and renal artery (Figure 5A and B) are dissected and encircled with vascular tapes. Gerota's fascia is opened and the perirenal fatty tissue is dissected off (Figure 6A) to expose the kidney and the protruding renal mass (Figure 6B). The line of excision for the renal lesion is scored on the renal capsule using monopolar curved scissors and cautery (Figure 7A). A barbed suture for performing renorraphy is introduced into the abdominal cavity and parked appropriately (Figure 7B).

An endoscopic bulldog clamp is prepared for use if needed. Although dissection of the renal hilum, including the renal artery and vein, might not be performed if this is going to increase operative time, it might be difficult for the assistant surgeon to apply a laparoscopic bulldog clamp in the event of unexpected bleeding while performing zero ischemia RPN.
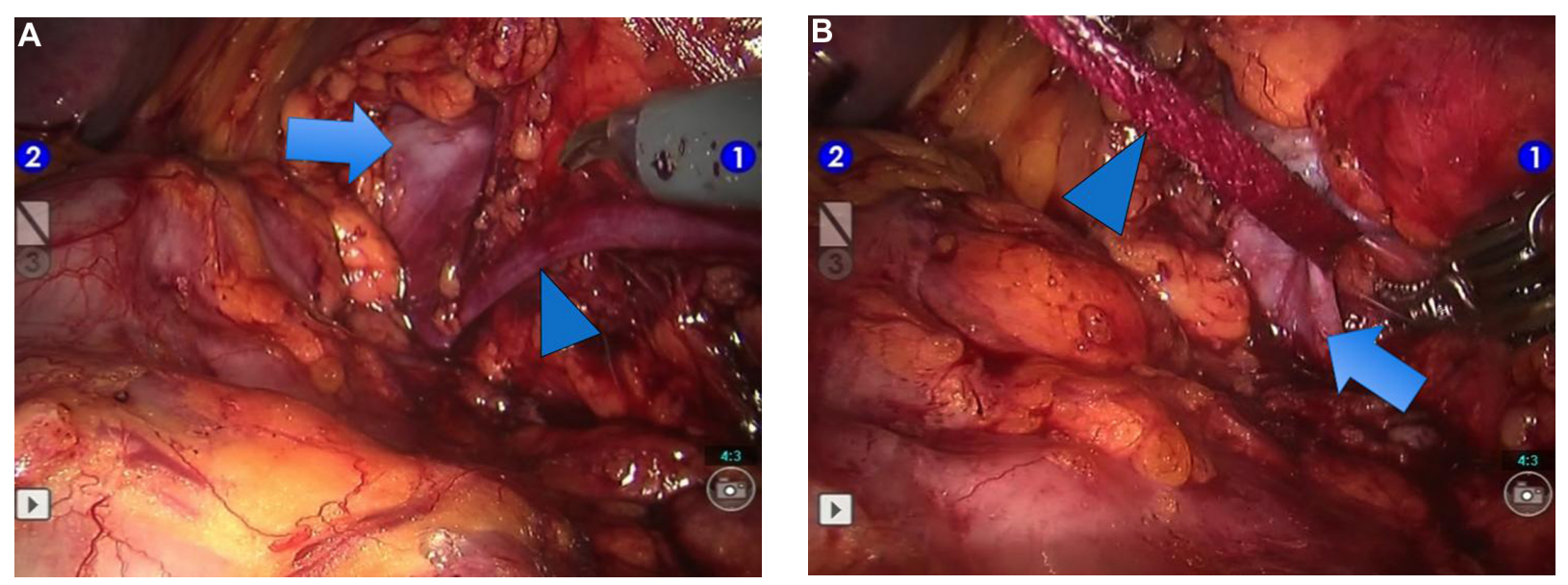

Figure 4 (A) Identification of renal vein (arrow). Arrowhead indicates gonadal vein. (B) Renal vein is dissected and encircled with a vascular tape (arrowhead). Arrow indicates renal vein.

Therefore, particularly when still becoming familiar with performing this procedure, we suggest dissecting the renal artery and vein and preparing them for clamping. Excision of the renal mass lesion is initiated using Maryland curved scissors and bipolar Maryland forceps with an adequate parenchymal margin, without applying an endoscopic bulldog clamp to the renal artery and vein. If no major bleeding is encountered, excision of the tumor can be completed (Figure $8 \mathrm{~A}-\mathrm{F}$ ). The intra-abdominal $\mathrm{CO}_{2}$ pressure may be increased if bleeding interferes with the excision procedure. Monopolar or bipolar cautery may also be used to control bleeding from the tumor bed. Intra-abdominal pressure might be increased initially when performing zero ischemia RPN if bleeding is greater than expected. Applying manual pressure might further decrease bleeding, and use of the fourth arm might provide additional assistance for the console surgeon in retracting tissues at the time of bleeding. Application of a laparoscopic bulldog clamp to the renal artery and vein would be expected to decrease bleeding, and the console surgeon should always keep in mind the option of converting to open surgery. Therefore, having adequate open surgical experience is essential when performing this type of surgery.

The zero ischemia RPN technique undoubtedly has some pitfalls, including a risk of major bleeding. To address this, we introduce a laparoscopic bulldog clamp into the abdominal cavity and locate it close to the predissected renal artery and vein before starting excision of the tumor. If major bleeding occurs, a bulldog clamp can be easily applied to the renal artery and vein. In this situation, the level of experience of the assistant surgeon is extremely important, so we suggest having available an assistant surgeon who has experience with this type of surgery.

A single absorbable barbed suture is used for internal and external renorraphy (Figure 9A-D). We use an absorbable

submit your manuscript | www.dovepress.com 

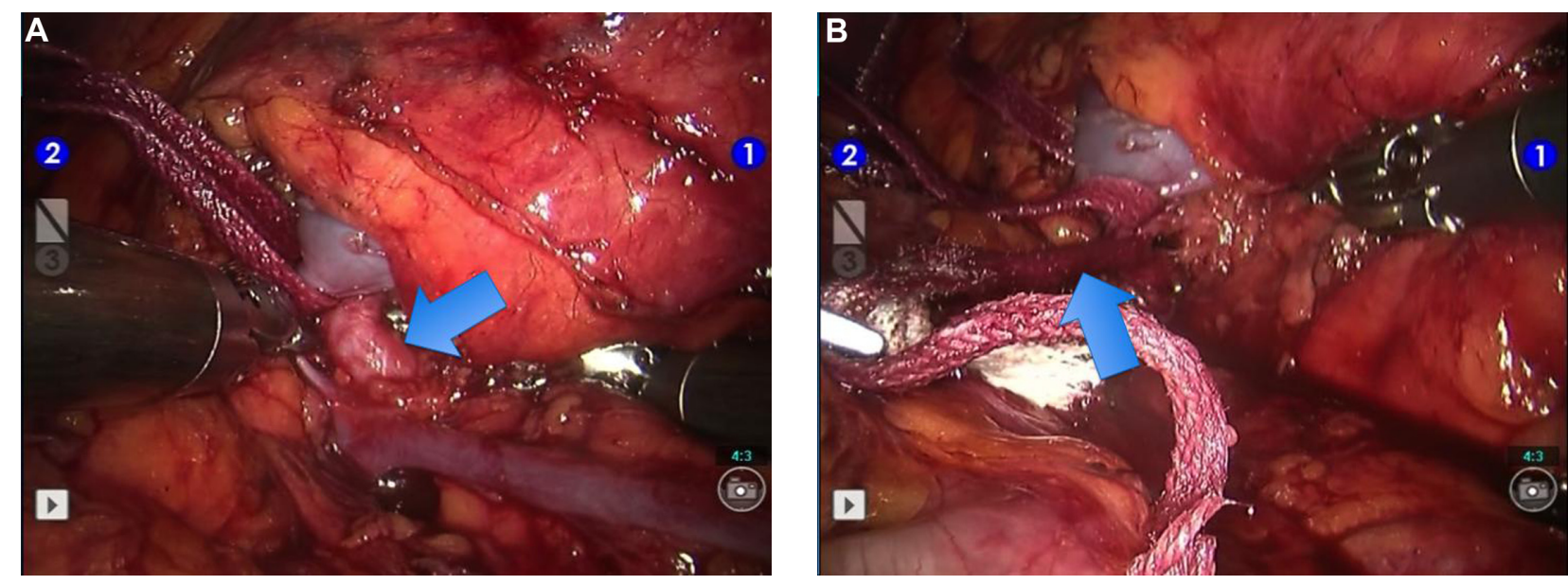

Figure 5 (A) Identification of renal artery (arrow). (B) Renal artery is dissected and encircled with a vascular tape (arrow).

polyglyconate barbed suture (3-0 V-Loc $180,45 \mathrm{~cm}$ with a $1 / 226 \mathrm{~mm}$ tapered needle; Covidien ${ }^{\mathrm{TM}}$, Mansfield, MA, USA) and initially perform internal renorraphy (Figure 9A). If the tumor bed is rather small, we proceed to external renorraphy using the same suture following completion of internal renorraphy (Figure 9B). Each barbed suture is kept tight with an absorbable endoclip on the renal capsule (Figure 9C). We prefer to use absorbable polyglactin suture clips (LAPRA-TY ${ }^{\circledR}$; Ethicon Endo-Surgery Inc., Cincinnati, $\mathrm{OH}, \mathrm{USA}$ ) by using their endoscopic clip applier that anchors and secures each single barbed suture strand on the renal capsule (Figure 9C and D). Thereafter, the excised renal mass is placed into the endobag (Figure 10A). After the vascular tapes are removed from the intra-abdominal cavity (Figure 10B), the perirenal fat over the completed partial nephrectomy site is closed using a $3 / 0$ polyglactin suture with an atraumatic needle (Figure 10C and D). The intraabdominal pressure is decreased to $5 \mathrm{mmHg}$ and the surgical

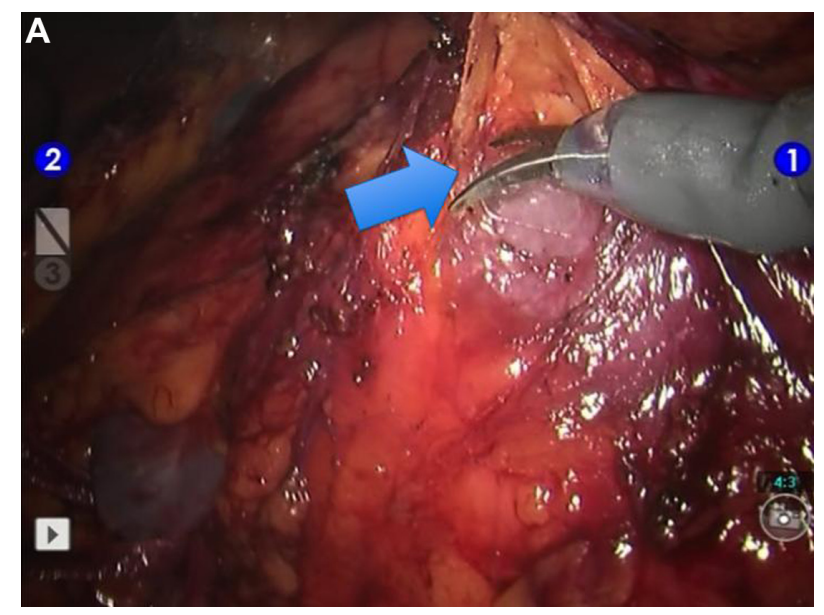

area is checked for bleeding. Following hemostasis, a drain is inserted into the abdominal cavity close to the surgical field. Abdominal ports are removed under direct vision and the port sites are closed.

\section{Discussion}

Although there are presently few publications on zero ischemia RPN in the literature, it is an attractive surgical procedure that enables excision of small selected renal masses with zero ischemia and without impairing overall renal function. ${ }^{3}$

Currently, we perform zero ischemia RPN in patients with small, peripherally located, exophytic renal mass lesions $<3 \mathrm{~cm}$ in size (Figure 6B). When such lesions are located in the lower kidney pole and an accessory lower pole artery is detected during robotic surgery, a laparoscopic bulldog clamp is applied selectively to the accessory artery so that the blood supply to the kidney overall is not impaired. More rarely, in the presence of an accessory artery

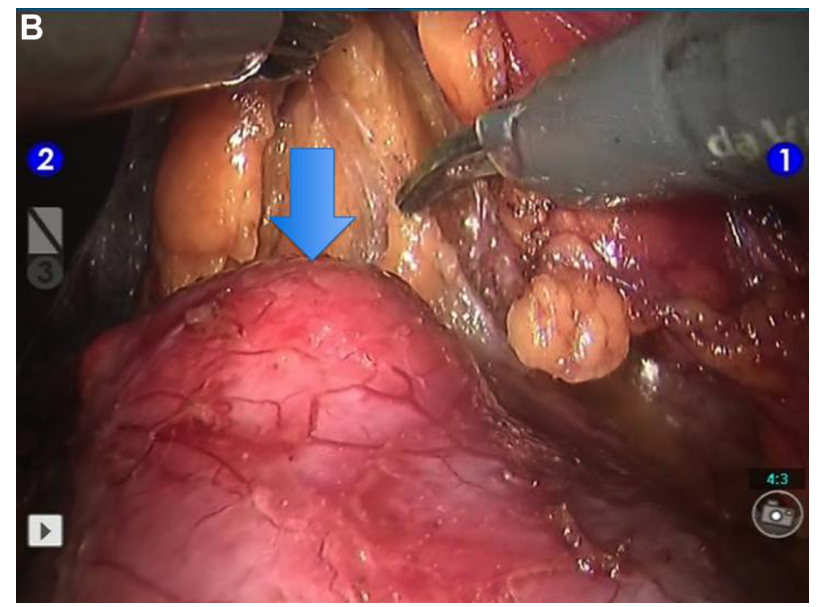

Figure 6 (A) Opening Gerota's fascia (arrow). (B) Identification of the renal mass lesion (arrow). 

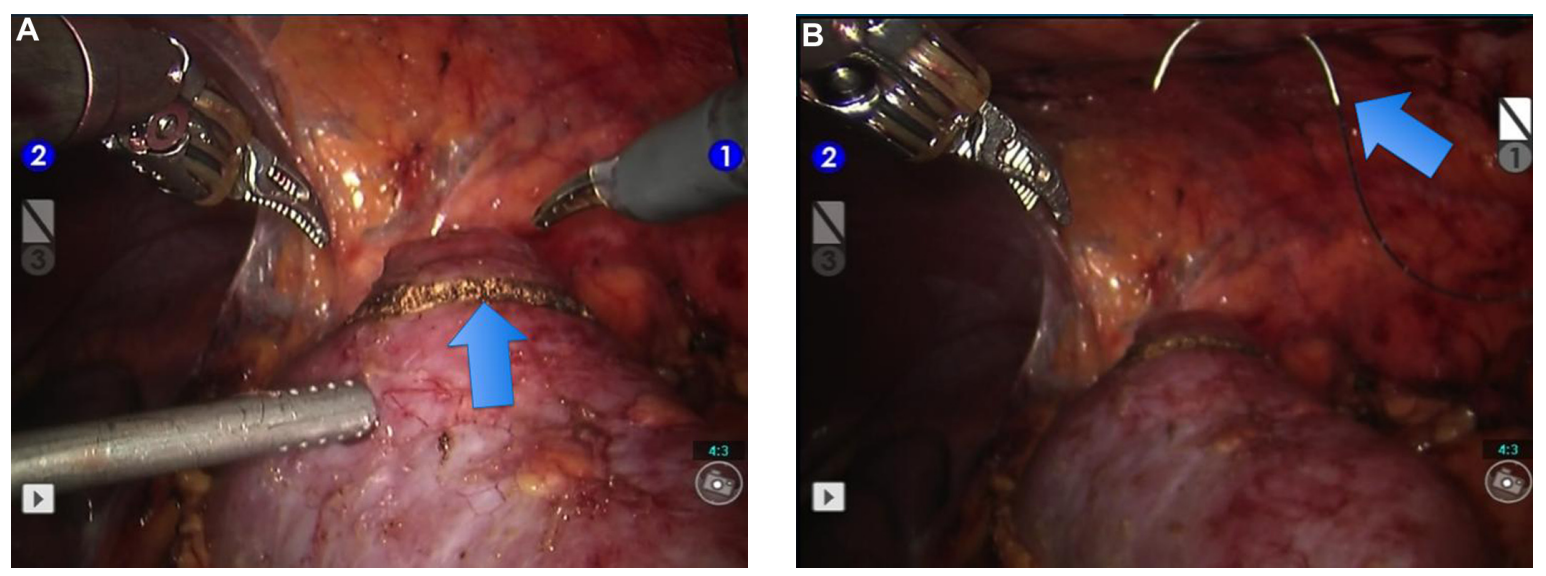

Figure 7 (A) Marking line of excision of the renal mass lesion on the renal capsule with monopolar cautery (arrow). (B) A barbed suture for performing renorraphy is introduced into the abdominal cavity (arrow).

selectively supplying the renal mass, a polymer ligation clip (Weck Hem-o-lok) might be applied to block this artery and thereby decrease the amount of bleeding during excision (Figure 11A-C). In both situations, the blood supply to the kidney overall would not be impaired because only selective clamping or cessation of blood supply to the mass is performed, which is a major advantage.

Temporary renal artery and/or renal vein clamping is performed before starting excision of the renal mass lesion in order to decrease bleeding and to provide a bloodless and
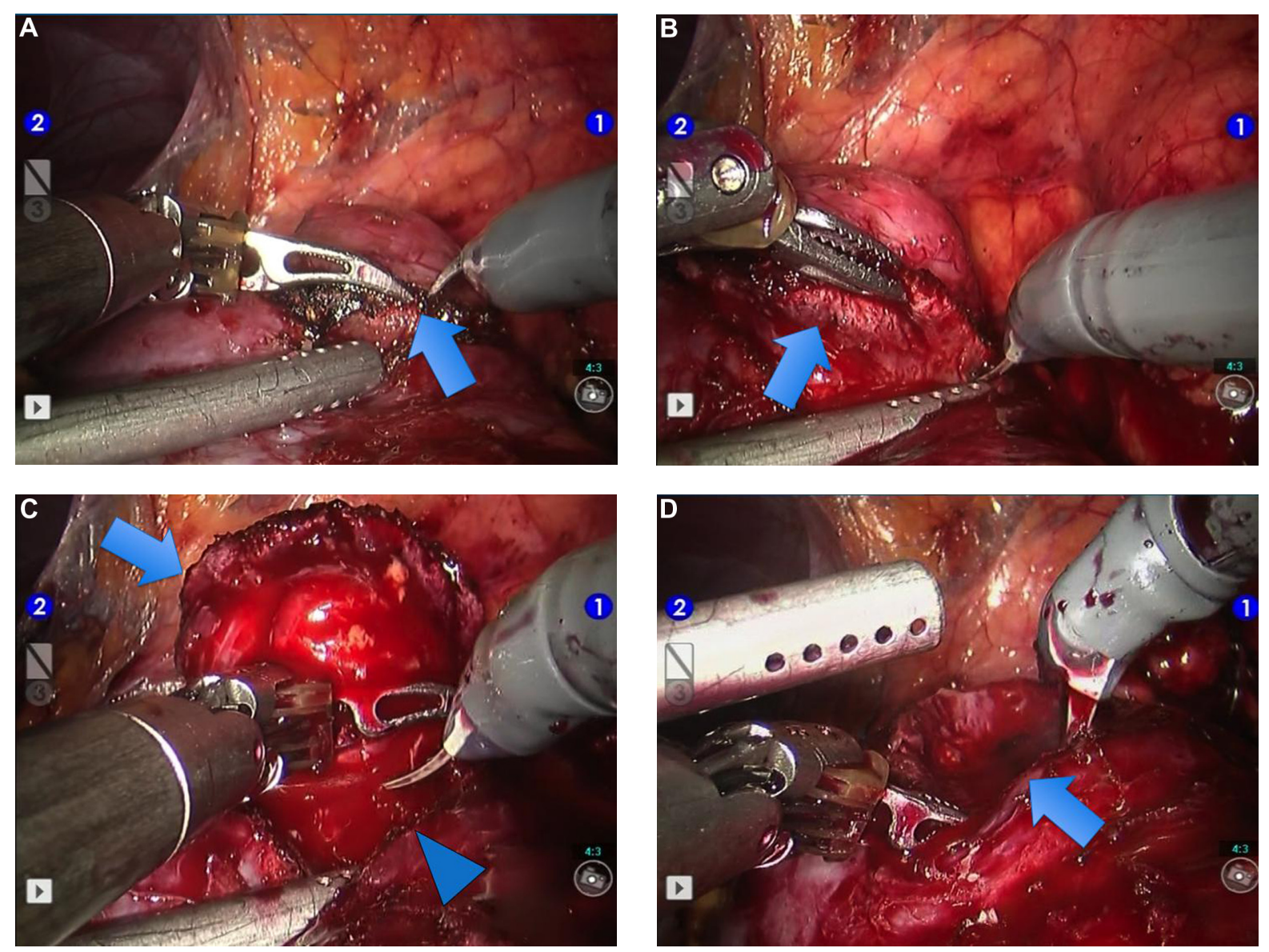

Figure 8 (A) Excision of the mass lesion using Maryland curved scissors and bipolar Maryland forceps (arrow). (B) Excision of the mass lesion using Maryland curved scissors and bipolar Maryland forceps and (arrow). (C) Further excision of the mass lesion using Maryland curved scissors and bipolar Maryland forceps (robotic zero ischemia partial nephrectomy is almost completed) (arrow and arrowhead). (D) Appearance of the tumor bed following excision of the tumor (arrow). Appearance of completed zero ischemia robotic partial nephrectomy and tumor bed. 

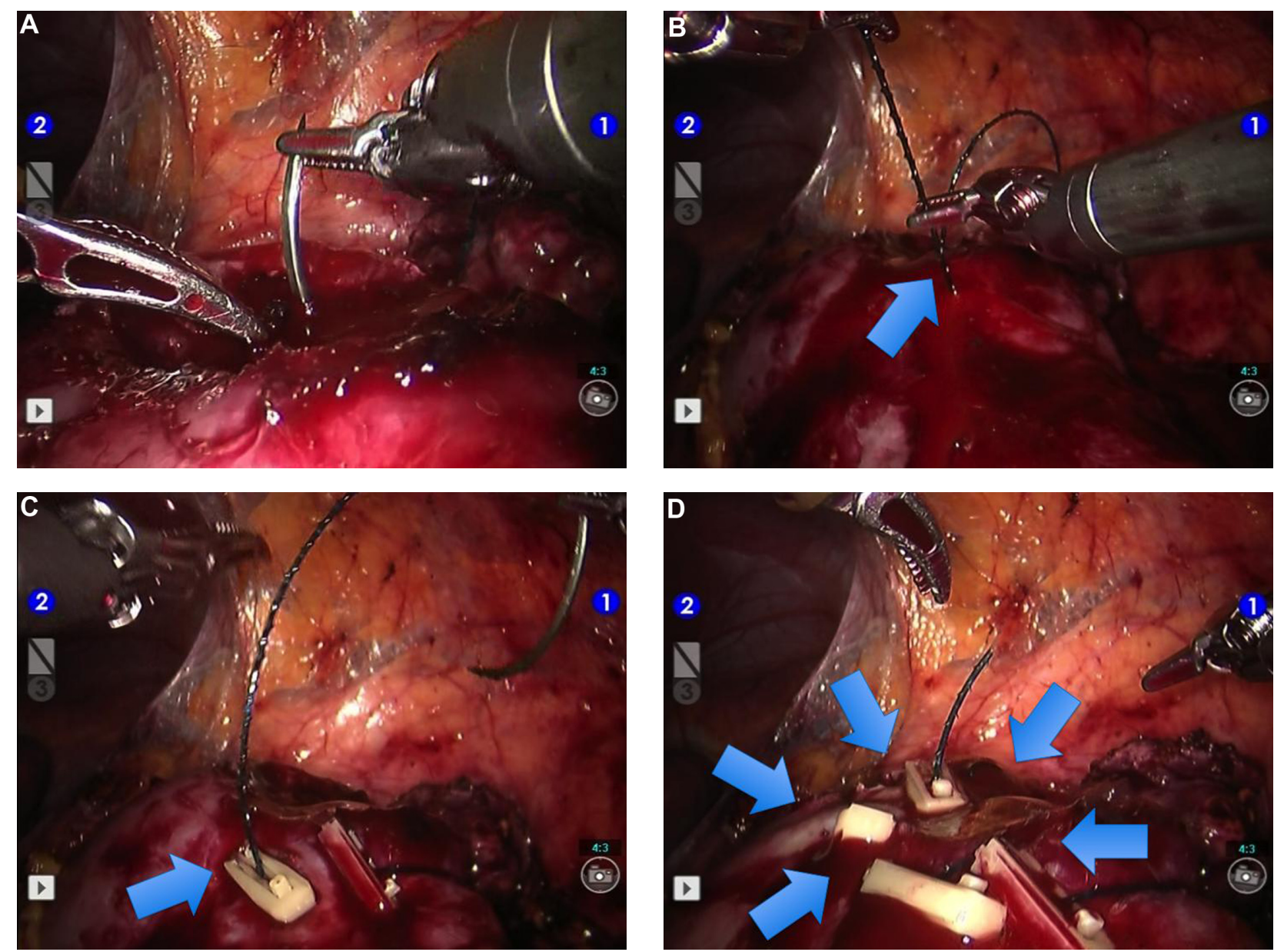

Figure 9 (A) Initiation of internal renorraphy using a barbed suture. (B) Proceeding to external renorraphy using the barbed suture following completion of internal renorraphy (arrow). (C) An absorbable polyglactin suture endoclip is applied using its endoscopic clip applier that anchors and secures each of a single barbed suture strand onto the renal capsule (arrow). (D) Appearance of the area on the kidney surface following completion of robotic partial nephrectomy and tumor bed renorraphy. Arrows indicate absorbable endoclips.

clear image for tissue dissection during RPN. ${ }^{3}$ However, hilar clamping halts the blood supply to the whole kidney, which may lead to a decrease in renal function, maintenance of which is particularly important in patients with underlying chronic kidney disease, ${ }^{3}$ and increase the need for dialysis in the future.

The robotic approach has the advantages of enabling more rapid tissue dissection, reconstruction, intracorporeal suturing, and antegrade double-J stenting if required. The Da Vinci-S four-arm surgical robotic system (Intuitive Surgical, Sunnyvale, CA, USA) has the advantages of threedimensional optical magnification, dexterity in motion, and the ability to perform tremor-free and delicate movements with three independent robotic arms in addition to the camera arm for the console surgeon. Therefore, in our experience, the technologic features of this surgical robotic system enable the operating console surgeon to perform zero ischemia or off-clamp RPN for selected small renal masses rapidly and safely without impairing overall renal function.

Currently, absorbable polyglyconate barbed sutures are being used during RPN and afford the console surgeon the opportunity to undertake continuous internal and external renorraphy (an important component of zero ischemia RPN) in a more rapid and safe way, thereby significantly facilitating the procedure. Hemostatic agents such as fibrin and tissue sealants may also be used for hemostasis during RPN if required.

Kaczmarek et al evaluated the perioperative and functional outcomes of RPN with $(n=283)$ and without $(n=49)$ hilar clamping in a recent, retrospective, multi-institutional study. ${ }^{3}$ Patients who underwent off-clamp RPN had a mean tumor size of $2.5 \pm 2.1 \mathrm{~cm}$. The off-clamp group had a significantly shorter mean operative time (156 minutes versus 185 minutes, $P<0.001$ ), greater estimated blood loss (228 $\mathrm{mL}$ versus $157 \mathrm{~mL}, P=0.009$ ), and a smaller decrease in 

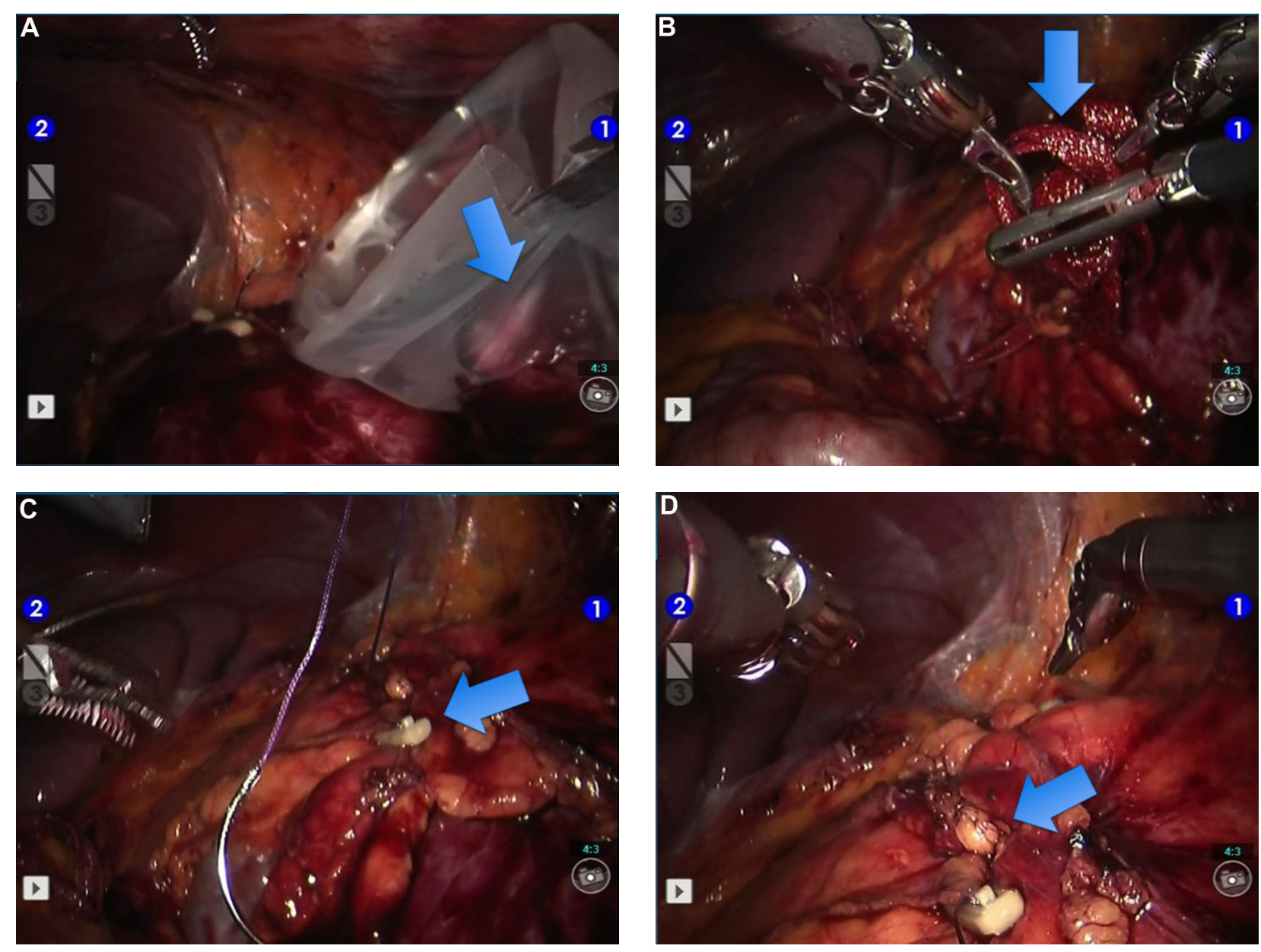

Figure 10 (A) Placement of the excised renal mass into the endobag (arrow). (B) Removal of the vascular tapes from the intra-abdominal cavity (arrow). (C) Closure of the perirenal fat over the area of completed partial nephrectomy using a Vicry ${ }^{\circledR}$ polyglactin suture (Ethicon Endo-Surgery Inc., Cincinnati, OH, USA) (arrow). (D) Appearance of completed robotic partial nephrectomy (arrow).

estimated glomerular filtration rate (eGFR, $2 \%$ versus $6 \%$, $P=0.008$ ). The authors concluded that zero ischemia RPN is a safe and feasible minimally invasive surgical option for appropriately selected patients with small renal masses. Adequate surgical experience reduces the risk of progression to renal insufficiency and enables better preservation of kidney function. ${ }^{3}$

The RENAL nephrometry score is used to categorize tumor complexity as low (4-6) or medium-high (7-12). ${ }^{5}$ Zero ischemia or off-clamp RPN is particularly recommended for less complex kidney tumors with low RENAL nephrometry scores. $^{3}$ The mean RENAL nephrometry score was $5.3 \pm 1.5$ in the study reported by Kaczmarek et al. ${ }^{3} \mathrm{Ng}$ et al described targeted vascular microdissection of renal artery branches in order to perform zero ischemia RPN. Their technique included preoperative computed tomography (CT) reconstruction of renal arterial branch anatomy and anatomic dissection of targeted tumor-specific tertiary or higher-order renal arterial branches. In addition, they suggested neurosurgical aneurysm microsurgical bulldog clamp(s) application for superselective tumor devascularization, and transient controlled reduction of blood pressure if necessary. ${ }^{6}$ They suggested that their technique can be successfully applied even in challenging medial and hilar tumors without hilar clamping, thereby avoiding renal ischemia during surgery. ${ }^{6}$ In their experience, mean estimated blood loss was $206 \mathrm{~mL}$, the mean hospital stay was 3.9 days, the mean operating time was 4.4 hours, and the mean postoperative transfusion rate was $21 \% .^{7}$ Ukimura et al developed a three-dimensional reconstruction method using $0.5 \mathrm{~mm}$ slice thickness $\mathrm{CT}$ scans to identify tumor-specific arterial branches and suggested that this method facilitates zero ischemia RPN without hilar cross clamping. ${ }^{8}$

Rao et al used CT with three-dimensional reconstruction to show renal arterial anatomy and its relationship to the renal mass. ${ }^{9}$ They used contrast-enhanced ultrasonography during the procedure, performed zero ischemia RPN with selective clamping, and demonstrated a nonperfused segment of kidney (occlusion angiography). They concluded that 

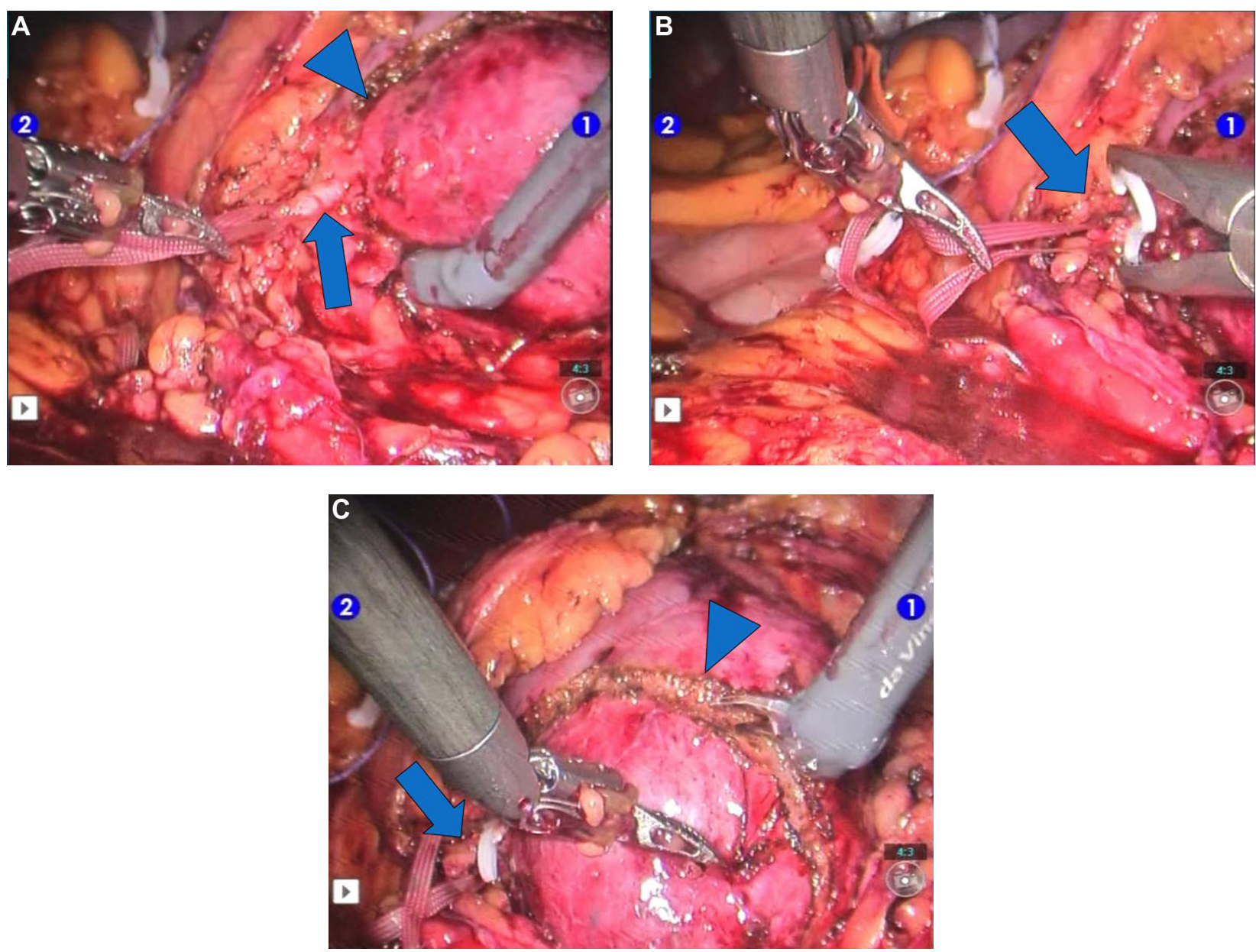

Figure I I (A) Appearance of the accessory artery (arrow) selectively supplying the small renal mass (arrowhead). (B) Appearance of a polymer ligation clip (arrow) applied on the accessory artery that is selectively supplying the mass. (C) Excision of the small renal mass (arrowhead) following application of the polymer ligation clip (arrow) to the accessory artery.

contrast-enhanced ultrasonography could be a useful tool for demonstrating if zero ischemia RPN is feasible by delineating the nonperfused region. ${ }^{9}$

In addition to zero ischemia RPN, George et al recently evaluated the perioperative outcomes of off-clamp versus complete hilar control laparoscopic partial nephrectomy cases in a series of 289 laparoscopic partial nephrectomies. Overall, 150 were performed off-clamp and rest were performed on-clamp. Tumors in the on-clamp group were significantly larger than those in the off-clamp group. Univariate analysis showed that eGFR was better preserved in the off-clamp group. This difference in eGFR was not significant at 6 months when warm ischemia time was limited to 30 minutes. They concluded that laparoscopic partial nephrectomy without hilar clamping is associated with less renal injury in terms of postoperative GFR. ${ }^{10}$

We perform zero ischemia RPN in patients with peripherally located kidney tumors that are less than $3 \mathrm{~cm}$ in size. Although we do not use intraoperative laparoscopic ultrasound in our practice, this might show the depth of the tumor, particularly those that are larger and complex, thereby facilitating tumor excision. However, intraoperative ultrasound is not essential for small and peripherally located small renal masses. With developing technology, robotic ultrasound probes are also being used. They allow optimal tumor identification and maximal autonomy with articulation of the robotic instrument. ${ }^{11}$

Near-infrared fluorescence imaging is another developing technologic tool that is reported to enable real-time intraoperative angiography and demonstrate selective ischemia, which might be useful in RPN. ${ }^{12}$ However, it is important to bear in mind that each additional technologic development used might increase the overall cost of the surgical procedure.

The effect of off-clamp partial nephrectomy on residual renal function has not been established as yet in the literature, although there are some data suggesting improved short-term and long-term preservation of renal function on eGFR. ${ }^{13}$ Off-clamp laparoscopic partial nephrectomy for T1b tumors 
has been suggested to be a feasible approach with respect to renal function and does not appear to carry an increased risk of transfusion despite increased blood loss. ${ }^{13}$ In their multiinstitutional robotic series, Kaczmarek et al suggested that offclamp RPN in the hands of high-volume surgeons preserves renal function, ${ }^{3}$ and detected a significantly smaller decrease in renal function using off-clamp RPN compared with RPN with hilar clamping. ${ }^{3}$ Likewise, Papalia et al evaluated the technical feasibility, safety, and functional outcomes of zero ischemia laparoscopic partial nephrectomy and RPN with controlled hypotension for renal tumors larger than $4 \mathrm{~cm}$ in size. ${ }^{14}$ The mean percentage decrease in effective renal plasma flow for the operated kidney was $1.8 \%$ in the off-clamp group $(n=51)$ versus $4.1 \%$ in the hilar clamping group $(n=43) .{ }^{14}$

In conclusion, renal cell carcinomas are currently detected incidentally as small renal masses, and surgical robotic technology has the advantages of enabling more rapid tissue dissection, reconstruction, intracorporeal suturing, threedimensional optical magnification, dexterity in motion, and ability to perform tremor-free and delicate movements with three independent robotic arms. For less complex, peripherally located, exophytic, and small renal masses $<3 \mathrm{~cm}$ in size, zero ischemic or off-clamp RPN seems to be a safe and feasible minimally invasive procedure that has the advantage of avoiding complete renal ischemia, which is particularly important in patients with underlying kidney disease.

\section{Disclosure}

The authors report no conflicts of interest in this work. AEC is a member of Robotic Urology Group, Young Academic Urologists (YAU) and the European Association of Urology (EAU).

\section{References}

1. Van Poppel H, Becker F, Cadeddu JA, et al. Treatment of localised renal cell carcinoma. Eur Urol. 2011;60(4):662-672.

2. Ficarra V, Minervini A, Antonelli A, et al. A multicenter matched-pair analysis comparing robot-assisted versus open partial nephrectomy. BJU Int. November 13, 2013. [Epub ahead of print.]

3. Kaczmarek BF, Tanagho YS, Hillyer SP, et al. Off-clamp robot-assisted partial nephrectomy preserves renal function: a multi-institutional propensity score analysis. Eur Urol. 2013;64(6):988-993.

4. Canda AE, Atmaca AF, Balbay MD. Robotic pyeloplasty: step by step surgical technique. Adv Robot Autom. 2013;2:1000111.

5. Kutikov A, Uzzo RG. The RENAL nephrometry score: a comprehensive standardized system for quantitating renal tumor size, location and depth. J Urol. 2009;182(3):844-853.

6. Ng CK, Gill IS, Patil MB, et al. Anatomic renal artery branch microdissection to facilitate zero-ischemia partial nephrectomy. Eur Urol. 2012;61(1):67-74.

7. Gill IS, Patil MB, Abreu AL, et al. Zero ischemia anatomical partial nephrectomy: a novel approach. $J$ Urol. 2012;187(3):807-814.

8. Ukimura O, Nakamoto M, Gill IS. Three-dimensional reconstruction of renovascular-tumor anatomy to facilitate zero-ischemia partial nephrectomy. Eur Urol. 2012;61(1):211-217.

9. Rao AR, Gray R, Mayer E, Motiwala H, Laniado M, Karim O. Occlusion angiography using intraoperative contrast-enhanced ultrasound scan (CEUS): a novel technique demonstrating segmental renal blood supply to assist zero-ischaemia robot-assisted partial nephrectomy. Eur Urol. 2013;63(5):913-919.

10. George AK, Herati AS, Srinivasan AK, et al. Perioperative outcomes of off-clamp vs complete hilar control laparoscopic partial nephrectomy. BJU Int. 2013;111(4 Pt B):E235-E241.

11. Kaczmarek BF, Sukumar S, Petros F, et al. Robotic ultrasound probe for tumor identification in robotic partial nephrectomy: initial series and outcomes. Int J Urol. 2013;20(2):172-176.

12. Bjurlin MA, Gan M, McClintock TR, et al. Near-infrared fluorescence imaging: emerging applications in robotic upper urinary tract surgery. Eur Urol. September 27, 2013. [Epub ahead of print.]

13. Kreshover JE, Kavoussi LR, Richstone L. Hilar clamping versus offclamp laparoscopic partial nephrectomy for T1b tumors. Curr Opin Urol. 2013;23(5):399-402.

14. Papalia R, Simone G, Ferriero M, et al. Laparoscopic and robotic partial nephrectomy without renal ischaemia for tumours larger than $4 \mathrm{~cm}$ : perioperative and functional outcomes. World $J$ Urol. 2012;3095):671-676.

\section{Publish your work in this journal}

Robotic Surgery: Research and Reviews is an international, peer reviewed, open access, online journal publishing original research, commentaries, reports, and reviews on the theory, use and application of robotics in surgical interventions. Articles on the use of supervisory-controlled robotic systems, telesurgical devices, and shared-control systems are

\section{Dovepress}

invited. The manuscript management system is completely online and includes a very quick and fair peer review system, which is all easy to use. Visit http://www.dovepress.com/testimonials.php to read real quotes from published authors. 